\title{
Associations of Snoring, Daytime Napping and Night Sleep Duration With Hyperuricemia in Rural Chinese Adults: the Henan Rural Cohort Study
}

\section{Xiaokang Dong}

Zhengzhou University

Xiaotian Liu

Zhengzhou University

Lulu Zhang

Zhengzhou University

Yan Wang

Zhengzhou University

\section{Runqi Tu}

Zhengzhou University

Wei Liao

Zhengzhou University

\section{Yikang Wang}

Zhengzhou University

\section{Ruiying Li}

Zhengzhou University

Jian Hou

Zhengzhou University

Zhenxing Mao

Zhengzhou University

Chongjian Wang

Zhengzhou University

Yuqian Li ( $\nabla$ liyuqian@zzu.edu.cn )

Zhengzhou University

\section{Research article}

Keywords: Snoring, Daytime napping, Night sleep duration, Hyperuricemia

Posted Date: August 17th, 2020

DOI: https://doi.org/10.21203/rs.3.rs-59400/v1 
License: (c) (i) This work is licensed under a Creative Commons Attribution 4.0 International License. Read Full License 


\section{Abstract}

Background. Evidence on the association of snoring, daytime napping or sleep duration with hyperuricemia (HUA) was limited, especially in the resources-poor areas. This study aimed to investigate the independent and joint effect of snoring frequency, daytime napping and sleep duration with prevalence of HUA in rural Chinese adults.

Methods. 29,643 participants aged 18-79 years were included from baseline survey of the Henan Rural Cohort Study. Sleep variables were assessed using the Pittsburgh Sleep Quality Index (PSQI). Multivariate logistic regression and linear regression models with HUA and serum uric acid level as dependent variables were conducted, respectively.

Results. Of the 29,643 included adults, 3498 suffered from HUA. Compared to never snoring, the adjusted odds ratio $(O R)$ and $95 \%$ confidence interval $(C l)$ of HUA for rare snoring, occasional snoring and habitual snoring were $1.35(1.17,1.56), 1.30(1.14,1.47)$ and $1.59(1.47,1.73)$, respectively. Compared with no napping, participants who had daytime napping of 61-90 and >91 min were associated with $29 \%$ and $30 \%$ increase in prevalence of HUA, respectively. But in night sleep duration groups, no significant associations were observed (all $P>0.05$ ). The positive associations between snoring and HUA were attenuated in older, female adults and those with chronic disease conditions. The joint of habitual snoring and longer daytime napping ( $\geq 61 \mathrm{~min}$ ) increased $63 \%$ prevalence on HUA.

Conclusion. Snoring or daytime napping may independently increase the prevalence of HUA and serum uric acid (SUA) level. Moreover, habitual snoring and longer daytime napping might be jointly associated with a higher prevalence of HUA.

Clinical Trial Registration The Henan Rural Cohort Study has been registered at Chinese Clinical Trial Register (Registration number: ChiCTR-OOC-15006699). http://www.chictr.org.cn/showproj.aspx? proj $=11375$

\section{Background}

Hyperuricemia (HUA), a condition characterized by abnormally elevated serum uric acid (SUA) levels, is the leading pathogenic factor in the development of gout [1] and potential risk factor for hypertension, type 2 diabetes mellitus (T2DM), and cardiovascular mortality [2-4]. The pooled prevalence of HUA in Chinese population from 2000 to 2014 is about 13.3\% [5], which is high and still increasing. Thus, special attention should be paid to the prevention of HUA and its influencing factors.

Sleep and sleep disorders are increasingly recognized as important factors of metabolic health. Sleepdisordered breathing, a condition characterized by hypopnea events or obstructive sleep apnea (OSA) during sleep, has been linked to multiple chronic metabolic diseases including HUA and gout [6-7]. As a surrogate marker for OSA, self-reported habitual snoring also related to many adverse health outcomes such as chronic kidney disease [8], T2DM [9], and metabolic syndrome [10]. However, the association 
between snoring and HUA was rarely reported, especially in the resource-poor areas. Only a recent study evaluated the cross-sectional association between snoring and SUA concentration and HUA in China [11], but it was limited in urban adults. Meantime, a growing number of epidemiological studies also have reported that longer sleep duration and daytime napping were independently or jointly associated with an increased prevalence of hyperglycemia, hypertension and incident heart failure and stroke [12-15], but the association of napping or sleep duration with HUA remain unclear. Additionally, as a sleep disorder marker, whether snoring can confound or modify the association between napping or sleep duration and HUA remains unknown. Thus, the joint association of napping or sleep duration and snoring with HUA also needs to be elucidated.

In recent years, the prevalence of HUA is high [16], meanwhile sleep-related problems such as poor sleep quality and longer napping duration were both prevalent in Chinese rural areas [17-18]. Given these and the scarcity of related data, it is of significant public health influence to explore the relationship of snoring, napping, sleep duration with HUA in rural Chinese population. Therefore, to address the data gap, this study investigated the independent and joint effect of snoring, daytime napping and night sleep duration with prevalence of HUA in rural Chinese adults.

\section{Methods}

\section{Study population}

A total of 39,259 subjects aged 18-79 years were recruited from Henan Rural Cohort Study, which was a prospective study focused on chronic non-communicable diseases. Detailed description of this cohort study design, methods, and participants recruitment have been previously published [19], and the cohort was registered in Chinese Clinical Trial Register (Registration number: ChiCTR-OOC-15006699). In short, the study was conducted in Yuzhou, Xinxiang, Tongxu, Yima and Suiping county of Henan Province, with the baseline survey has been completed from July 2015 to October 2017. For the current analysis, after excluding participants who were missing data on SUA level $(n=54)$, had malignant tumor and serious renal disease $(n=350)$, and those with incomplete information about sleep data $(n=9212), 29,643$ adults $(12,128$ men and 17,515 women) were included in final. The protocol of this study was approved by the Zhengzhou University Life Science Ethics Committee. Informed consent was obtained from all participants.

\section{Assessment of potential covariates}

The potential covariates including demographic covariates, lifestyle factors and the family history of disease were collected by well-trained staffs using a standardized questionnaire. Demographic covariates included age, gender (male or female), education level ("primary school or below" or "middle school or above"), marital status ("married/cohabitating" or "unmarried/divorced/widowed") and per capita monthly income ("<500 RMB", "500-1000 RMB" or " $\geq 1000$ RMB"). Lifestyle factors included smoking status, drinking status, physical activity and dietary pattern. Smoking status was classified into never, current, and former groups. Drinking status also was classified into never, current, and former groups. 
Physical activity was divided into three levels (low, moderate and high) based on the International Physical Activity Questionnaire (IPAQ) [20]. Dietary pattern was categorized four types as previously by using a standard principal component analysis method [21], dietary pattern I with a high intake of red meat, white meat and fish; pattern II with a high intake of vegetables, staple food, and fruits; pattern III with a high intake of grains, nuts, beans, pickles and animal oils; and pattern $₫$ with milk and eggs. In addition, obesity (yes/no), hypertension (yes/no), T2DM (yes/no) and dyslipidemia status (yes/no) were assessed as previous definitions [16], family history of gout (yes/no) also was obtained.

\section{Assessment of sleep variables}

Sleep quality and related sleep variables were assessed using the Pittsburgh Sleep Quality Index (PSQI), which is a validated self-report questionnaire consisting of 19 elements to assess sleep quality and disturbances [22]. The Chinese version PSQI used by us has been widely used to assess sleep quality with good overall reliability ( $r=0.82-0.83)$ and test-retest reliability $(r=0.77-0.85)$ [23]. For the snoring frequency, participants were asked two questions (1) Do you know, or have you ever heard that you snore? (yes or no) and (2) How often do you snore during the past month? (never, rarely snore $<1$ day per week, occasionally for 1-2 days per week, habitually $\geq 3$ days per week) [24]. Daytime napping was assessed by asking participants "Did you take a nap usually over the past year?" Those who answered yes were further asked to report the average daytime napping duration. Napping duration was reclassified into 0 min (reference), 1-30 min, 31-60 min, 61-90 $\mathrm{min}$ and $\geq 91 \mathrm{~min}$ [18]; in addition, self-reported night sleep duration also was determined based on answers to the questions: "On average how many hours actual sleep duration did you get at night during the past month?" Night Sleep duration was grouped as $<5,5-6,6-7,7-8$ (reference), 8-9, 9-10, and $\geq 10$ hours [25].

\section{Definition of HUA}

Blood samples were collected from individual antecubital vein after at least $8 \mathrm{~h}$ of overnight fasting to measure multiple biochemical indicators. SUA level was measured by ROCHE Cobas C501 automatic biochemical analyzer with enzymatic colorimetric method. Definition of HUA was determined as SUA level $>417 \mu \mathrm{mol} / \mathrm{L}(7.0 \mathrm{mg} / \mathrm{dL})$ and $>357 \mu \mathrm{mol} / \mathrm{L}(6.0 \mathrm{mg} / \mathrm{dL})$ for men and women, respectively [15].

\section{Statistical analysis}

All analyses were conducted using IBM SPSS V.19.0 and R 3.5.0. Descriptive statistics included one-way analyses of variance and chi-squared tests were conducted to compare the differences of baseline characteristics according to the HUA status of participants, including demographic and socio-economic characteristics, lifestyle risk factors, and sleeping variables. Multivariable logistic regression models were performed to explore the associations between sleep variables (snoring frequency, daytime napping and night sleep duration) and prevalence of HUA. Interactions were tested by adding interaction terms of these sleep variables by pairwise combination, respectively. Moreover, multivariable linear regression analyses were conducted to evaluate the relationship of these sleep variables with SUA level. Three models were constructed: model 1, age-, sex-adjusted model; model 2, adjusted for age, gender, education 
level, marital status, average monthly income, smoking status, drinking status, physical activity and dietary pattern; model 3, model 2 plus adjustment for snoring, daytime napping and night sleep duration where applicable.

Based on the results of logistic regression analyses, the subgroup analyses were further conducted to examine whether the significant associations between snoring or daytime napping (each $30 \mathrm{~min}$ increment) and prevalence of HUA were modified by age, gender, obesity, hypertension, T2DM and dyslipidemia status. To test the robustness of the results, sensitivity analyses also were performed to repeat the regression analyses by gender or additionally adjusting for family history of gout, obesity, T2DM, hypertension and dyslipidemia conditions. A P-value $<0.05$ was considered to be statistically significant.

Furthermore, snoring frequency, daytime napping and night sleep duration also were recoded as never, rarely ( $<1$ day/week), occasionally ( $1-2$ days/week), and habitually ( $\geq 3$ days/week) and $0 \mathrm{~min}, 1-30 \mathrm{~min}$, 31-60 min, and $\geq 61$ min and $<7,7-8,8-9$ and $\geq 9$ hours for combined effects, where the combined never snoring and no napping group, never snoring and night sleep duration (7-8 hours), and no napping and night sleep duration (7-8 hours), served as the reference categories, respectively.

\section{Results}

\section{Characteristics of study participants}

Among the 29643 participants, 3498 participants (11.80\%) with HUA were identified (Table 1). In general, compared with non-HUA, those with HUA comprised a greater proportion of men, younger individuals. Besides, participants with HUA had higher education level and per capita monthly income, were more likely to smoke and drink, lower physical activity, had higher snoring frequency and longer daytime napping duration than those without HUA (all $P<0.001$ ). 
Table 1

Basic characteristics of study participants by with and without hyperuricemia.

\begin{tabular}{|c|c|c|c|}
\hline \multirow[t]{2}{*}{ Characteristics } & \multicolumn{3}{|l|}{ Total $(n=29643)$} \\
\hline & Non-HUA $(n=26145)$ & HUA $(n=3498)$ & $P$-value \\
\hline Age (year, mean $\pm S D)$ & $55.64 \pm 12.20$ & $53.07 \pm 13.58$ & $<0.001$ \\
\hline Gender (n, \%) & & & $<0.001$ \\
\hline Men & $10362(39.63)$ & $1766(50.49)$ & \\
\hline Women & $15783(60.37)$ & $1732(49.51)$ & \\
\hline Education level (n, \%) & & & $<0.001$ \\
\hline Primary school or below & 11707 (44.77) & $1410(40.31)$ & \\
\hline Middle school or above & $14438(55.23)$ & 2088 (59.69) & \\
\hline Marital status (n, \%) & & & 0.303 \\
\hline Married/cohabitating & $23584(90.20)$ & $3136(89.65)$ & \\
\hline Unmarried/divorced/widowed & $2561(9.80)$ & $362(10.35)$ & \\
\hline Per capita monthly income $(n, \%)$ & & & $<0.001$ \\
\hline$<500 \mathrm{RMB}$ & $9452(36.15)$ & $1228(35.11)$ & \\
\hline 500-1000 RMB & $8324(31.84)$ & $1021(29.19)$ & \\
\hline$\geq 1000 \mathrm{RMB}$ & $8369(30.01)$ & $1249(35.71)$ & \\
\hline Smoking status (n, \%) & & & $<0.001$ \\
\hline Never & $19010(72.71)$ & $2296(65.63)$ & \\
\hline Former & $1999(7.65)$ & $340(9.72)$ & \\
\hline Current & $5136(19.64)$ & $862(24.64)$ & \\
\hline Drinking status $(\mathrm{n}, \%)$ & & & $<0.001$ \\
\hline Never & $20691(78.86)$ & $2370(67.75)$ & \\
\hline Former & $1292(4.94)$ & $168(4.80)$ & \\
\hline Current & $4234(16.19)$ & $960(27.44)$ & \\
\hline Physical activity (n, \%) & & & $<0.001$ \\
\hline Low & $8146(31.16)$ & $1286(36.76)$ & \\
\hline
\end{tabular}

a $P$ Values were derived from analysis of variance or Mann-Whitney $U$ tests for continuous variables according to data distribution and $\chi^{2}$ tests for category variables. 


\begin{tabular}{|c|c|c|c|}
\hline \multirow[t]{2}{*}{ Characteristics } & \multicolumn{3}{|l|}{ Total $(n=29643)$} \\
\hline & Non-HUA ( $n=26145)$ & HUA $(n=3498)$ & $P$-value ${ }^{a}$ \\
\hline Moderate & $9660(36.95)$ & $1231(35.19)$ & \\
\hline High & 8339 (31.90) & $981(28.04)$ & \\
\hline Snoring frequency $(n, \%)$ & & & $<0.001$ \\
\hline Never & $15373(58.80)$ & $1678(47.97)$ & \\
\hline Rarely (< 1 day/week) & $1549(5.92)$ & $247(7.06)$ & \\
\hline Occasionally ( $1-2$ days/week) & $2365(9.05)$ & $346(9.89)$ & \\
\hline Habitually ( $\geq 3$ days/week) & $6858(26.23)$ & $1227(35.08)$ & \\
\hline Daytime napping (min, mean \pm SD) & $58.21 \pm 51.41$ & $64.34 \pm 52.72$ & $<0.001$ \\
\hline Night sleep duration ( $h /$ day, mean $\pm S D$ ) & $7.70 \pm 1.32$ & $7.70 \pm 1.31$ & 0.952 \\
\hline Dietary pattern $(n, \%)$ & & & $<0.001$ \\
\hline Pattern I & $5442(23.90)$ & $951(27.19)$ & \\
\hline Pattern $\otimes$ & $5656(26.36)$ & $719(20.55)$ & \\
\hline Pattern $\otimes$ & $8297(28.19)$ & $1035(29.59)$ & \\
\hline Pattern $\otimes$ & $6750(21.55)$ & $793(22.67)$ & \\
\hline
\end{tabular}

\section{Independent associations of three sleep variables with prevalence of HUA}

In the three models, snoring frequency and daytime napping were all significantly associated with the increased prevalence of HUA, but no significant associations could be observed between night sleep duration and HUA (Table 2). According to model 3, compared with never snoring, the adjusted odds ratio $(O R)$ and $95 \%$ confidence interval $(C l)$ of HUA for rare snoring, occasional snoring and habitual snoring were $1.35(1.17,1.56), 1.30(1.14,1.47)$ and $1.59(1.47,1.73)$. The $O R(95 \% C l)$ of snoring was $1.49(1.38$, 1.60) compared to non-snorers. Compared with no napping, participants who had daytime napping of 61-90 and >91 min were associated with $29 \%$ and $30 \%$ increase in prevalence of HUA, respectively. Each 30 min increment in napping duration was associated with a 7\% elevated risk of HUA. In addition, sensitivity analyses by gender and additionally adjusting for family history of gout, obesity, T2DM, hypertension and dyslipidemia conditions did not substantially change these estimates (Additional file 1: Table S1). 
Table 2

Snoring, daytime napping, night sleep duration and prevalence of hyperuricemia.

\begin{tabular}{|c|c|c|c|c|}
\hline \multirow[t]{2}{*}{ Sleep variables } & \multirow{2}{*}{$\begin{array}{l}\text { Number } \\
\text { (Prevalence, \%) }\end{array}$} & \multicolumn{3}{|l|}{$O R(95 \% C l)$} \\
\hline & & Model 1 & Model 2 & Model 3 \\
\hline \multicolumn{5}{|l|}{$\begin{array}{l}\text { Snoring frequency } \\
\text { (day/week) }\end{array}$} \\
\hline Never & $17051(9.84)$ & 1 (Ref.) & 1 (Ref.) & 1 (Ref.) \\
\hline Rarely (< 1 day/week) & 1796 (13.75) & $\begin{array}{l}1.36(1.17 \\
1.57)\end{array}$ & $\begin{array}{l}1.35(1.17 \\
1.57)\end{array}$ & $\begin{array}{l}1.35(1.17, \\
1.56)\end{array}$ \\
\hline $\begin{array}{l}\text { Occasionally (1-2 } \\
\text { days/week) }\end{array}$ & $2711(12.76)$ & $\begin{array}{l}1.29(1.14 \\
1.46)\end{array}$ & $\begin{array}{l}1.30(1.15 \\
1.47)\end{array}$ & $\begin{array}{l}1.30(1.14, \\
1.47)\end{array}$ \\
\hline $\begin{array}{l}\text { Habitually ( } \geq 3 \\
\text { days/week) }\end{array}$ & $8085(15.18)$ & $\begin{array}{l}1.59(1.47 \\
1.73)\end{array}$ & $\begin{array}{l}1.59(1.47, \\
1.73)\end{array}$ & $\begin{array}{l}1.59(1.47, \\
1.73)\end{array}$ \\
\hline Snoring & 12592 & $\begin{array}{l}1.49(1.39 \\
1.60)\end{array}$ & $\begin{array}{l}1.49(1.38, \\
1.60)\end{array}$ & $\begin{array}{l}1.49(1.38, \\
1.60)\end{array}$ \\
\hline \multicolumn{5}{|l|}{ Daytime napping (min) } \\
\hline 0 & 8387 (10.37) & 1 (Ref.) & 1 (Ref.) & 1 (Ref.) \\
\hline $1-30$ & $3393(11.52)$ & $\begin{array}{l}1.10(0.98 \\
1.26)\end{array}$ & $\begin{array}{l}1.08(0.95 \\
1.23)\end{array}$ & $\begin{array}{l}1.08(0.95, \\
1.23)\end{array}$ \\
\hline $31-60$ & $8148(11.16)$ & $\begin{array}{l}1.05(0.95 \\
1.16)\end{array}$ & $\begin{array}{l}1.05(0.95 \\
1.15)\end{array}$ & $\begin{array}{l}1.04(0.94, \\
1.15)\end{array}$ \\
\hline $61-90$ & $2647(13.71)$ & $\begin{array}{l}1.32(1.16, \\
1.51)\end{array}$ & $\begin{array}{l}1.31(1.14, \\
1.49)\end{array}$ & $\begin{array}{l}1.29(1.13, \\
1.48)\end{array}$ \\
\hline$\geq 91$ & 7068 (13.65) & $\begin{array}{l}1.32(1.20 \\
1.46)\end{array}$ & $\begin{array}{l}1.31(1.19 \\
1.45)\end{array}$ & $\begin{array}{l}1.30(1.18, \\
1.44)\end{array}$ \\
\hline Each 30 min increment & & $\begin{array}{l}1.07(1.05 \\
1.10)\end{array}$ & $\begin{array}{l}1.07(1.05 \\
1.10)\end{array}$ & $\begin{array}{l}1.07(1.05, \\
1.10)\end{array}$ \\
\hline \multicolumn{5}{|l|}{$\begin{array}{l}\text { Night sleep duration } \\
\text { (hours) }\end{array}$} \\
\hline$<5$ & $969(12.28)$ & $\begin{array}{l}1.12(0.92, \\
1.37)\end{array}$ & $\begin{array}{l}1.09(0.89 \\
1.34)\end{array}$ & $\begin{array}{l}1.11(0.90, \\
1.36)\end{array}$ \\
\hline $5-6$ & $1189(12.03)$ & $\begin{array}{l}1.03(0.85 \\
1.24)\end{array}$ & $\begin{array}{l}1.02(0.84, \\
1.23)\end{array}$ & $\begin{array}{l}1.01(0.84, \\
1.22)\end{array}$ \\
\hline
\end{tabular}

Model 1, adjusted for age, gender; multivariable model 2 adjusted for age, gender, education level, marital status, average monthly income, smoking status, drinking status, physical activity, dietary pattern; model 3 plus adjustment for snoring, daytime napping and night sleep duration where applicable. 


\begin{tabular}{|c|c|c|c|c|}
\hline \multirow[t]{2}{*}{ Sleep variables } & \multirow{2}{*}{$\begin{array}{l}\text { Number } \\
\text { (Prevalence, \%) }\end{array}$} & \multicolumn{3}{|l|}{ OR $(95 \% C l)$} \\
\hline & & Model 1 & Model 2 & Model 3 \\
\hline $6-7$ & $5420(11.88)$ & $\begin{array}{l}1.03(0.93 \\
1.15)\end{array}$ & $\begin{array}{l}1.04(0.93, \\
1.15)\end{array}$ & $\begin{array}{l}1.04(0.94, \\
1.15)\end{array}$ \\
\hline $7-8$ & 10446 (11.53) & 1 (Ref.) & 1 (Ref.) & 1 (Ref.) \\
\hline $8-9$ & 7835 (12.05) & $\begin{array}{l}1.08(0.99 \\
1.19)\end{array}$ & $\begin{array}{l}1.08(0.99, \\
1.18)\end{array}$ & $\begin{array}{l}1.08(0.99, \\
1.19)\end{array}$ \\
\hline $9-10$ & $2886(11.75)$ & $\begin{array}{l}1.08(0.95 \\
1.23)\end{array}$ & $\begin{array}{l}1.07(0.94, \\
1.22)\end{array}$ & $\begin{array}{l}1.08(0.94, \\
1.23)\end{array}$ \\
\hline$\geq 10$ & 898 (11.69) & $\begin{array}{l}1.10(0.88, \\
1.36)\end{array}$ & $\begin{array}{l}1.05(0.85, \\
1.30)\end{array}$ & $\begin{array}{l}1.04(0.84, \\
1.29)\end{array}$ \\
\hline \multicolumn{5}{|c|}{$\begin{array}{l}\text { Model 1, adjusted for age, gender; multivariable model } 2 \text { adjusted for age, gender, education level, } \\
\text { marital status, average monthly income, smoking status, drinking status, physical activity, dietary } \\
\text { pattern; model } 3 \text { plus adjustment for snoring, daytime napping and night sleep duration where } \\
\text { applicable. }\end{array}$} \\
\hline
\end{tabular}

\section{Association between three sleep variables and SUA level}

In Table 3, positive associations were also found between snoring frequency or daytime napping and SUA level. After adjusting multiple variables in model 3 , those participants with snoring were associated with a $13.30 \mu \mathrm{mol} / \mathrm{L}$ higher SUA level $(95 \% \mathrm{Cl}, 11.59,15.01)$ compared with never snoring. Meantime, each $30 \mathrm{~min}$ increment in daytime napping was associated with a $2.87 \mu \mathrm{mol} / \mathrm{L}$ higher SUA level $(95 \% \mathrm{Cl}, 2.31$, 3.42). But the associations of night sleep duration with SUA level were nonsignificant. Similarly, these results remained materially unchanged in sensitivity analyses in which the models were reassessed by gender or additionally adjusting for family history of gout, obesity, T2DM, hypertension and dyslipidemia conditions (Additional file 1: Table S2). 
Table 3

Adjusted $\beta$ and $95 \% \mathrm{Cl}$ of serum uric acid level in relation to snoring frequency, daytime napping and night sleep duration.

\section{Sleep variables \\ Snoring frequency
(day/week)}

Number $\quad \beta(95 \% C l)$

Model 1

Model 2

Model 3

$\begin{array}{llll}\text { Never } & 17051 & 0 \text { (Ref.) } & 0 \text { (Ref.) }\end{array}$

Rarely (< 1 day/week) $\quad 1796 \quad 9.17(5.59,12.75) \quad 9.02(5.47,12.58) \quad 8.83(5.59,12.75)$

Occasionally

$2711 \quad 8.19(5.21,11.18) \quad 8.41(5.44,11.38) \quad 8.32(5.21,11.18)$

(1-3 days/week)

Habitually

8085

16.19 (14.22,

17.17)

16.19 (14.22,

18.17)

16.06 (14.22,

( $\geq 4$ days/week)

Snoring

$13.42(11.70$
$15.14)$

13.43 (11.72,

15.14)

13.30 (11.59,

15.01)

\section{Daytime napping (min)}

\begin{tabular}{|c|c|c|c|c|}
\hline 0 & 8387 & 0 (Ref.) & 0 (Ref.) & 0 (Ref.) \\
\hline $1-30$ & 3393 & $4.03(1.09,6.97)$ & $2.69(-0.24,5.62)$ & $2.44(-0.48,5.36)$ \\
\hline $31-60$ & 8148 & $5.40(3.14,7.65)$ & $4.94(2.70,7.19)$ & $4.76(2.53,7.00)$ \\
\hline $61-90$ & 2647 & $\begin{array}{l}10.31(7.08 \\
13.54)\end{array}$ & $9.81(6.61,13.02)$ & $9.41(6.21,12.60)$ \\
\hline$\geq 91$ & 7068 & $\begin{array}{l}11.91(9.57 \\
14.25)\end{array}$ & $\begin{array}{l}11.53(9.20 \\
13.86)\end{array}$ & $\begin{array}{l}11.28(8.97 \\
13.60)\end{array}$ \\
\hline Each 30 min increment & & $2.98(2.43,3.54)$ & $2.93(2.38,3.49)$ & $2.87(2.31,3.42)$ \\
\hline \multicolumn{5}{|l|}{$\begin{array}{l}\text { Night sleep duration } \\
\text { (hours) }\end{array}$} \\
\hline$<5$ & 969 & $3.43(-1.43,8.29)$ & $2.74(-2.09,7.58)$ & $3.61(-1.20,8.42)$ \\
\hline $5-6$ & 1189 & $1.63(-2.80,6.05)$ & $1.51(-2.89,5.91)$ & $1.49(-2.89,5.86)$ \\
\hline $6-7$ & 5420 & $1.26(-1.16,3.69)$ & $1.38(-1.02,3.79)$ & $1.50(-0.89,3.89)$ \\
\hline $7-8$ & 10446 & 1 (Ref.) & 1 (Ref.) & 1 (Ref.) \\
\hline
\end{tabular}

Model 1, adjusted for age, gender; multivariable model 2 adjusted for age, gender, education level, marital status, average monthly income, smoking status, drinking status, physical activity, dietary pattern; model 3 plus adjustment for snoring, daytime napping and night sleep duration where applicable. 


\begin{tabular}{|lclll|}
\hline Sleep variables & Number & $\boldsymbol{\beta}(\mathbf{9 5 \%} \boldsymbol{C I})$ & \\
\cline { 2 - 5 } & & Model 1 & Model 2 & Model 3 \\
\hline $\mathbf{8 - 9}$ & 7835 & $0.94(-1.22,3.10)$ & $0.92(-1.23,3.07)$ & $0.99(-1.15,3.13)$ \\
\hline $\mathbf{9 - 1 0}$ & 2886 & $-0.69(-3.74,2.36)$ & $-0.85(-3.89,2.18)$ & $-0.61(-3.63,2.41)$ \\
\hline$\geq 10$ & 898 & $-1.55(-6.59,3.50)$ & $-2.91(-7.93,2.12)$ & $-3.03(-8.03,1.97)$ \\
\hline $\begin{array}{l}\text { Model 1, adjusted for age, gender; multivariable model 2 adjusted for age, gender, education level, } \\
\text { marital status, average monthly income, smoking status, drinking status, physical activity, dietary } \\
\text { pattern; model 3 plus adjustment for snoring, daytime napping and night sleep duration where } \\
\text { applicable. }\end{array}$ \\
\hline
\end{tabular}

\section{Subgroup analysis}

The results of the associations between snoring or napping (each 30 min increment) and HUA in subgroup stratified by age, gender, and chronic disease conditions (obesity, T2DM, hypertension, and dyslipidemia conditions) are presented in Figs. 1 and 2. The $O R$ of HUA relating to snoring was significant lower among those older participants, women, or those with obesity, T2DM, hypertension, and dyslipidemia conditions (Fig. 1). But for the association between napping and HUA in Fig. 2, significant difference can be found in most subgroups, the association was only insignificant in participants aged < $35(P=0.495)$ and those with T2DM $(P=0.162)$.

\section{Joint associations of sleep variables with HUA}

In Fig. 3. A and Additional file 1: Table S3, the joint analysis of snoring frequency and daytime napping in total population showed that compared with reference (never snoring and napping group), participants with combined habitually snore ( $\geq 3$ days/week) and daytime napping $\geq 61 \mathrm{~min}$ had the highest prevalence of HUA (OR 1.63; $95 \% \mathrm{Cl}=1.41,1.88)$. Similarly, as shown in Additional file 1: Figure $\mathrm{S} 1$ and Table S4, further analysis stratified by gender also found the joint associations were consistently exist in both men and women, with the corresponding highest risks were $1.66(1.33,2.07)$ and $1.61(1.32,1.97)$, respectively. But for the combined analyses of snoring frequency and night sleep duration or daytime napping and night sleep duration, no significant strong associations can be consistently found in total population (Fig. 3. B and C) and in men and women (Additional file 1: Table S4-8, Figure S2-3).

\section{Discussion}

In the large sample study, we observed that both snoring frequency and daytime napping were independently associated with increased prevalence of HUA and elevated SUA level. In addition, subgroup analysis showed that the positive associations between snoring and HUA were attenuated in older, female participants and those with chronic disease conditions. More importantly, a joint effect of habitual napping and longer daytime napping ( $\geq 61 \mathrm{~min}$ ) increased 63\% prevalence for HUA. To our knowledge, 
this study is the first one exploring the independent and joint association between snoring and daytime napping with HUA in rural Chinese adults.

Some existing studies indicated that patients with sleep apnea have a high blood uric acid levels and prevalence of HUA [26-29]. In a large epidemiological sample in Brazil, a strong association was found between uric acid levels and OSA even after adjustment for confounding factors such as gender, age, BMI, social class, ethnicity, cholesterol, triglycerides, blood pressure and glucose [26]. Another study conducted in overweight children and adolescents demonstrated a relationship between the severity of sleep apnea and increased levels of SUA, independent of abdominal adiposity [27]. Pływaczewski et al. has found that HUA is frequent in both males and females with OSA [28-29]. In addition, recent several studies also demonstrated sleep apnea is independently associated with an increased risk of incident gout [29], which is a common inflammatory arthritis caused by HUA. As a highly prevalent condition associated with OSA and sleep disturbance in bed partners, snoring is commonly considered as a mild form of sleep apnea or a useful screening tool for OSA [11, 31]. However, studies about the association between soring and SUA level or HUA were limited. A recent study found that self-reported habitual snoring was associated with higher SUA concentration, but it was only conducted in Chinese urban adults [11].

The current study found that snoring was independently associated with increased SUA level and risk of HUA in rural Chinese adults, which addressed the data gap in rural area. Meanwhile, the positive associations between snoring and HUA were attenuated in participants with chronic disease conditions (obesity, T2DM, hypertension and dyslipidemia). In accordance with our findings, a previous study also found the association between snoring and HUA was attenuated in participants with some clinical outcomes such as depression, diabetes, hypertension, and high-cholesterol levels [31]. Although the association was attenuated, it still remained significant after further adjustment of these chronic disease conditions (Additional file 1: Table S1), which suggested those habitual snorers may be related with these chronic diseases. In addition, snoring may play an independently important role in the development of HUA [11]. The biologic mechanisms linking snoring to the HUA were unclear. Some main hypotheses were that habitual snoring is usually accompanied by OSA. First, OSA-induced hypoxemia can cause a rise in adenosine triphosphate (ATP) degradation which eventually increases purine concentrations, leading to elevated blood uric acid level [32]. Second, excretion of lactic acid, generated during the hypoxic episodes in OSA, could result in a higher renal reabsorption of uric acid [33].

In this study, we also observed that daytime napping was independently associated with increased SUA level and risk of HUA. Many studies have reported that daytime napping or sleep duration was associated with metabolic diseases [12-15]. But few studies have evaluated the relationship of sleep variables such as daytime napping and night sleep duration with HUA. Only a survey from the National Health and Nutrition Examination indicated a positive relationship of multiple sleep variables including snoring, snorting, and daytime sleepiness, with prevalence of HUA [34], meantime night sleep duration was not associated with HUA, which is consistent with our results. However, the effect of napping on HUA was not explored in the above-mentioned survey. As the first study to explore association between napping and 
HUA in Chinese rural adults, we also further found the joint effect of snoring frequency and napping duration on HUA. Some previous study had found the combined effect of napping or sleep quality and night sleep duration on some metabolic diseases $[15,35]$. Similarly, in the current study, we observed that habitual snoring ( $\geq 3$ days/week) combined with longer daytime napping ( $\geq 61 \mathrm{~min}$ ) increased $63 \%$ risk of HUA in total participants ( $66 \%$ for men and $63 \%$ for men), though no significant joint effects of snoring frequency and night sleep duration or daytime napping and night sleep duration existed. Biological mechanisms underlying the joint effects are not clear, but this emphasizes the adverse health consequences of snoring and daytime napping, as well as jointly predicted HUA prevalence. We speculated that snoring as a sleep disorder marker may partly contribute the adverse effects of longer daytime napping on HUA. In addition, the exact mechanism underlying the association between the napping and HUA also remains unclear. One potential biological pathway is through inflammation, as Janna et al reported that increased napping is an independent predictor of inflammation in adults [36]. Previous animal studies also have shown that intermittent hypoxia and resultant oxygen desaturation may be associated with subsequent activation of inflammatory pathways and cause elevated SUA level [37]. Further studies are needed to clarify the mechanism.

The strengths of this study are as follows. Firstly, the current study thoroughly discussed the independent association between snoring frequency, daytime napping, night sleep duration and HUA based on a large rural cohort, which help us fill this knowledge gap about the relationship in rural Chinese population.

Secondly, to our knowledge, this is the first study to found the joint effect of snoring and napping on HUA, which both were prevalent in Chinese rural area and should be given more attention. However, several limitations need to be noted: first, because of the cross-sectional nature, we could not establish the causal relationship of snoring or napping or night sleep duration with HUA. Thus, further prospective studies are demanded to confirm the results from this study. Second, data on snoring frequency and napping were obtained from a self-reported questionnaire rather than an accurate evaluation using polysomnography. Nevertheless, similar assessment of these variables has been widely used [35, 38].

Third, because our participants hardly reported the use of sleep medications, we also did not include this variable in the current study.

\section{Conclusions}

In conclusion, snoring frequency or daytime napping might be important predictors of high SUA level and HUA prevalence among rural Chinese adults. Moreover, habitual snoring combined with longer daytime napping ( $\geq 61 \mathrm{~min}$ ) may be associated with a higher prevalence of HUA. Intervention of snoring and measures to control longer daytime napping could contribute to reducing prevalence of HUA and SUA level, which further control the development of gout.

\section{Abbreviations}


Adenosine triphosphate; Cl:Confidence intervals; HUA:Hyperuricemia; IPAQ:International Physical Activity Questionnaire; OR:odds ratio serum uric acid; OSA:obstructive sleep apnea; PSQI:Pittsburgh Sleep Quality Index; SUA:serum uric acid; T2DM:Type 2 diabetes mellitus.

\section{Declarations}

\section{Acknowledgements}

The authors thank all of the participants, coordinators, and administrators for their support and help during the research.

\section{Funding}

This research was supported by the Foundation of National Key Program of Research and Development of China (Grant NO: 2016YFC0900803), National Natural Science Foundation of China (Grant NO: 81573243, 81602925), Henan Provincial Science Fund for Distinguished Young Scholars (Grant NO: 164100510021), Science and Technology Innovation Talents Support Plan of Henan Province Colleges and Universities (Grant NO: 14HASTIT035). The funders had no role in the study design, data collection and analysis, decision to publish, or preparation of the manuscript.

\section{Availability of data and materials}

The datasets used during the current study are available from the corresponding author upon reasonable request. Please contact with Yuqian Li about detail information. Email address: liyuqian@zzu.edu.cn

\section{Author contributions}

During the research, Yuqian Li designed the study. Xiaokang Dong, Xiaotian Liu, Lulu Zhang, Yan Wang, Runqi Tu, Wei Liao, Yikang Wang, Ruiying Li, Jian Hou, Zhenxing Mao, Chongjian Wang and Yuqian Li directed the collection of the data. Xiaokang Dong and Xiaotian Liu analyzed the data. Xiaokang Dong and Xiaotian Liu wrote the manuscript. Tanko Abdulai, Xiaotian Liu provided writing assistance. All authors read and approve this version of the article.

\section{Ethics approval and consent to participate}

The study complies with the Declaration of Helsinki, ethics approval was obtained from the "Zhengzhou University Life Science Ethics Committee", and written informed consent was obtained for all participants. Ethic approval code: [2015] MEC (S128).

\section{Consent for publication}

Not applicable.

\section{Competing interests}


The authors declare that they have no competing interests.

\section{Author details}

a Department of Epidemiology and Biostatistics, College of Public Health, Zhengzhou University, Zhengzhou, Henan, PR China.

${ }^{b}$ Department of Clinical Pharmacology, School of Pharmaceutical Science, Zhengzhou University, Zhengzhou, Henan, PR China.

\section{References}

1. Kuo CF, Grainge MJ, Zhang W, Doherty M. Global epidemiology of gout: prevalence, incidence and risk factors. Nature reviews. Rheumatology. 2015; 11:649-62.2.

2. Kuwabara M, Niwa K, Nishi Y, Mizuno A, Asano T, Masuda K, et al. Relationship between serum uric acid levels and hypertension among Japanese individuals not treated for hyperuricemia and hypertension. Hypertension research: official journal of the Japanese Society of Hypertension. 2014; 37:785-9.

3. Bandaru P, Shankar A. Association between Serum Uric Acid Levels and Diabetes Mellitus. International journal of endocrinology. 2011:604715.

4. Chen JH, Chuang SY, Chen HJ, Yeh WT, Pan WH. Serum uric acid level as an independent risk factor for all-cause, cardiovascular, and ischemic stroke mortality: a Chinese cohort study. Arthritis and rheumatism.2009; 61:225-32.

5. Liu R, Han C, Wu D, Xia X, Gu J, Guan H, et al. Prevalence of Hyperuricemia and Gout in Mainland China from 2000 to 2014: A Systematic Review and Meta-Analysis. BioMed research international. 2015:762820.

6. Punjabi NM, Newman AB, Young TB, Resnick HE, Sanders MH. Sleep-disordered breathing and cardiovascular disease: an outcome-based definition of hypopneas. Am J Respir Crit Care Med. 2008; 177:1150-1155.

7. Zhang Y, Peloquin CE, Dubreuil M, Roddy E, Lu N, Neogi T, et al. Sleep Apnea and the Risk of Incident Gout: A Population-Based, Body Mass Index-Matched Cohort Study. Arthritis \& rheumatology (Hoboken, N.J.). 2015; 67:3298-302.

8. Song J, Wang C, Ma A, Zheng H, Zheng W, Hou X, et al. Self-reported snoring is associated with chronic kidney disease independent of metabolic syndrome in middle-aged and elderly Chinese. $\mathrm{J}$ Diabetes Investig. 2019; 10:124-30.

9. Strand LB, Carnethon M, Biggs ML, Djoussé L, Kaplan RC, Siscovick DS, et al. Sleep Disturbances and Glucose Metabolism in Older Adults: The Cardiovascular Health Study. Diabetes care. 2015; 38:2050-8. 
10. Shin MH, Kweon SS, Choi BY, Kim MK, Chun BY, Shin DH, et al. Self-reported snoring and metabolic syndrome: the Korean Multi-Rural Communities Cohort Study. Sleep \& breathing = Schlaf \& Atmung. 2014; 18:423-30.

11. Xiong X, He F, Sun G, Li Y, Shi Y, Ge X, et al. The relationship between self-reported habitual snoring and hyperuricemia among Chinese urban adults: a cross-sectional study. Sleep medicine. 2010; 68:207-12.

12. Izci Balserak B, Jackson N, Ratcliffe SA, Pack Al, Pien GW. Sleep-disordered breathing and daytime napping are associated with maternal hyperglycemia. Sleep \& breathing = Schlaf \& Atmung.2013; 17:1093-102.

13. Cao Z, Shen L, Wu J, Yang H, Fang W, Chen W, et al. The effects of midday nap duration on the risk of hypertension in a middle-aged and older Chinese population: a preliminary evidence from the TongjiDongfeng Cohort Study, China. J Hypertens. 2014; 32:1993-8; discussion 98.

14. Wannamethee SG, Papacosta O, Lennon L, Whincup PH. Self-Reported Sleep Duration, Napping, and Incident Heart Failure: Prospective Associations in the British Regional Heart Study. Journal of the American Geriatrics Society.2016; 64:1845-50.

15. Zhou L, Yu K, Yang L, Wang H, Xiao Y, Qiu G, et al. Sleep duration, midday napping, and sleep quality and incident stroke: The Dongfeng-Tongji cohort. Neurology. 2020; 94: e345-e56.

16. Dong X, Zhang H, Wang F, Liu X, Yang K, Tu R, et al. Epidemiology and prevalence of hyperuricemia among men and women in Chinese rural population: The Henan Rural Cohort Study. Modern rheumatology. 2019;1-11.

17. Wang Y, Li Y, Liu X, Liu R, Mao Z, Tu R, et al. Gender-specific prevalence of poor sleep quality and related factors in a Chinese rural population: the Henan Rural Cohort Study. Sleep Med. 2019;54:134141.

18. Liu R, Li Y, Wang F, Liu X, Zhou H, Wang P, et al. Age- and gender-specific associations of napping duration with type 2 diabetes mellitus in a Chinese rural population: the RuralDiab study. Sleep Med. 2017;33:119-124.

19. Liu X, Mao Z, Li Y, Wu W, Zhang X, Huo W, et al. Cohort Profile: The Henan Rural Cohort: a prospective study of chronic non-communicable diseases. Int J Epidemiol. 2019; 48(6):1756-1756j.

20. Bennie JA, Chau JY, van der Ploeg HP, Stamatakis E, Do A, Bauman A. The prevalence and correlates of sitting in European adults - a comparison of 32 Eurobarometer-participating countries. Int J Behav Nutr Phys Act. 2013;10:107.

21. Wang B, Liu L, Qiao D, Xue Y, Liu X, Zhang D, et al. The association between frequency of away-from home meals and type 2 diabetes mellitus in rural Chinese adults: the Henan Rural Cohort Study. Eur J Nutr. 2020;19.

22. Buysse DJ, Reynolds CF 3rd, Monk TH, Berman SR, Kupfer DJ. The Pittsburgh Sleep Quality Index: a new instrument for psychiatric practice and research. Psychiatry Res. 1989;28(2):193-213.

23. Tsai PS, Wang SY, Wang MY, Su CT, Yang TT, Huang CJ, et al. Psychometric evaluation of the Chinese version of the Pittsburgh Sleep Quality Index (CPSQI) in primary insomnia and control 
subjects. Qual Life Res. 2005;14(8):1943-52.

24. Zhang H, Li S, Chen G, Abdulai T, Liu X, Wang Y, et al. Ambient air pollutants aggravate association of snoring with prevalent hypertension: results from the Henan Rural Cohort. Chemosphere. 2020;256:127108.

25. Zhang H, Zhao X, Li Y, Mao Z, Huo W, Jiang J, et al. Night sleep duration and sleep initiation time with hypertension in Chinese rural population: the Henan Rural Cohort. Eur J Public Health. 2020; 30(1):164-170.

26. Hirotsu C, Tufik S, Guindalini C, Mazzotti DR, Bittencourt LR, Andersen ML. Association between uric acid levels and obstructive sleep apnea syndrome in a large epidemiological sample. PLoS One. 2013;8(6):e66891.

27. Verhulst SL, Van Hoeck K, Schrauwen N, Haentjens D, Rooman R, Van Gaal L, et al. Sleep-disordered breathing and uric acid in overweight and obese children and adolescents. Chest. 2007;132(1):76-80.

28. Pływaczewski R, Bednarek M, Jonczak L, Górecka D, Sliwińiski P. Hiperurykemia u meiczyzn chorych na obturacyjny bezdech senny (obs) [Hyperuricaemia in males with obstructive sleep apnoea (osa)]. Pneumonol Alergol Pol. 2005;73(3):254-9.

29. Pływaczewski R, Bednarek M, Jonczak L, Górecka D, Sliwiński P. Hiperurykemia u kobiet chorych na obturacyjny bezdech senny [Hyperuricaemia in females with obstructive sleep apnoea]. Pneumonol Alergol Pol. 2006;74(2):159-65.

30. van Durme C, Spaetgens B, Driessen J, Nielen J, Sastry M, Boonen A, et al. Obstructive sleep apnea and the risk of gout: a population-based case-control study. Arthritis Res Ther. 2020; 22(1):92.

31. Sowho M, Sgambati F, Guzman M, Schneider H, Schwartz A. Snoring: A Source of Noise Pollution and Sleep Apnea Predictor. Sleep. 2019; Dec 14:zsz305. doi: 10.1093/sleep/zsz305.

32. Saito H, Nishimura M, Shibuya E, Makita H, Tsujino I, Miyamoto K, et al. Tissue hypoxia in sleep apnea syndrome assessed by uric acid and adenosine. Chest. 2002; 122(5):1686-94.

33. Kanbay A, Inonu H, Solak Y, Erden A, Uslu E, Yuksel SA, et al. Uric acid as a potential mediator of cardiovascular morbidity in obstructive sleep apnea syndrome. Eur J Intern Med. 2014;25(5):471476.

34. Wiener RC, Shankar A. Association between Serum Uric Acid Levels and Sleep Variables: Results from the National Health and Nutrition Survey 2005-2008. Int J Inflam. 2012;2012:363054.

35. Liu R, Li Y, Mao Z, Liu X, Zhang H, Yang K, et al. Gender-specific independent and combined doseresponse association of napping and night sleep duration with type 2 diabetes mellitus in rural Chinese adults: the RuralDiab study. Sleep Med. 2018; 45:106-113.

36. Mantua J, Spencer RM. The interactive effects of nocturnal sleep and daytime naps in relation to serum C-reactive protein. Sleep Med. 2015;16(10):1213-6.

37. Sahebjani H. Changes in urinary uric acid excretion in obstructive sleep apnea before and after therapy with nasal continuous positive airway pressure. Chest. 1998 Jun;113(6):1604-8. 
38. Endeshaw Y, Rice TB, Schwartz AV, Stone KL, Manini TM, Satterfield S, et al. Snoring, daytime sleepiness, and incident cardiovascular disease in the health, aging, and body composition study. Sleep. 2013;1;36(11):1737-45.

\section{Figures}

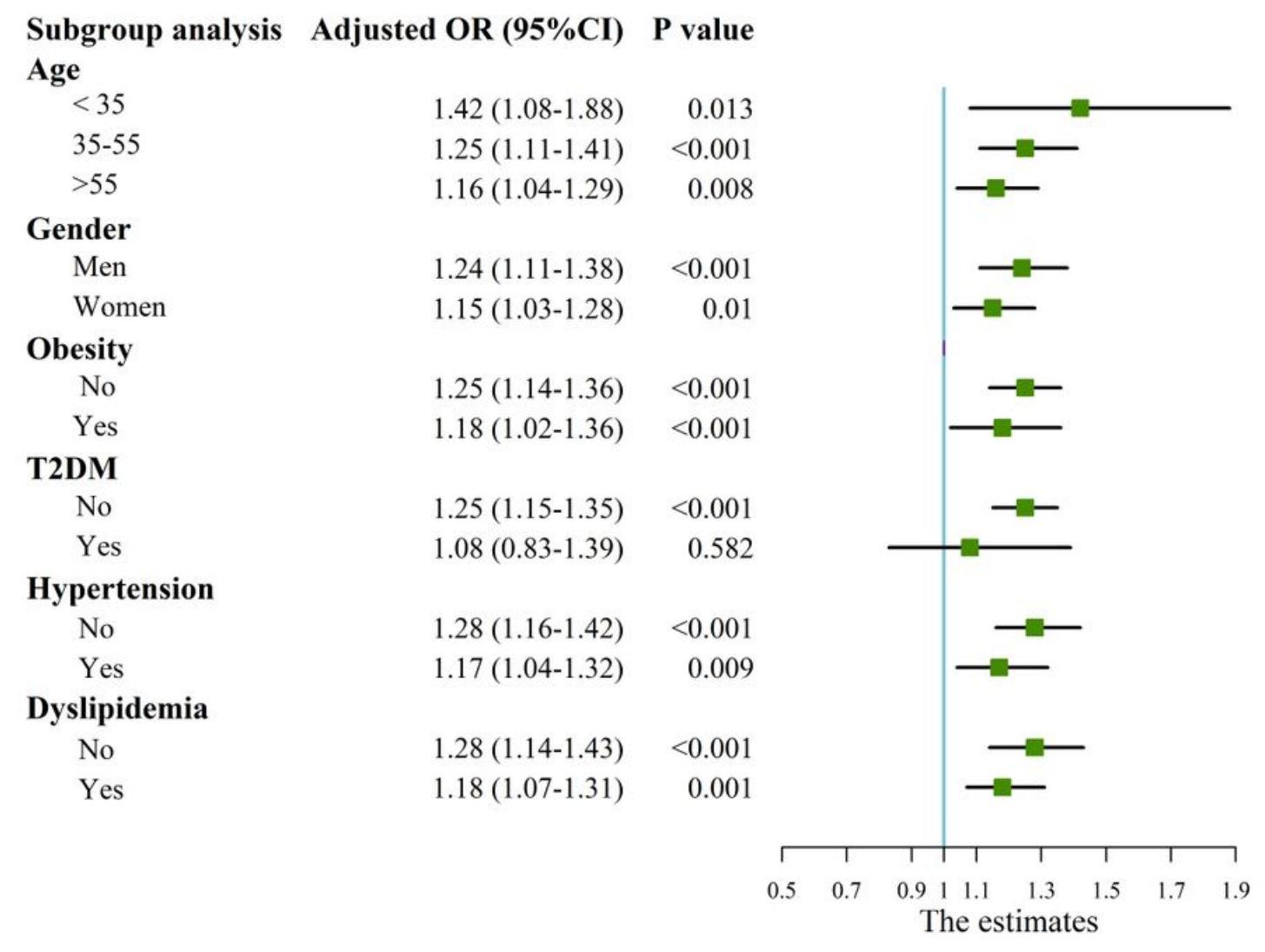

\section{Snoring vs never snoring}

\section{Figure 1}

Snoring (vs never snoring) and prevalence of hyperuricemia, stratified by age, gender, and chronic disease conditions. Adjusted for age, gender, education level, marital status, average monthly income, smoking status, drinking status, physical activity, dietary pattern, daytime napping, night sleep duration, family history of gout, obesity, T2DM, hypertension and dyslipidemia conditions. Each group adjusted for the other covariates except itself. OR= Odds Ratio, $\mathrm{Cl}=$ confidence interval, $\mathrm{T} 2 \mathrm{DM}=$ type 2 diabetes mellitus. 


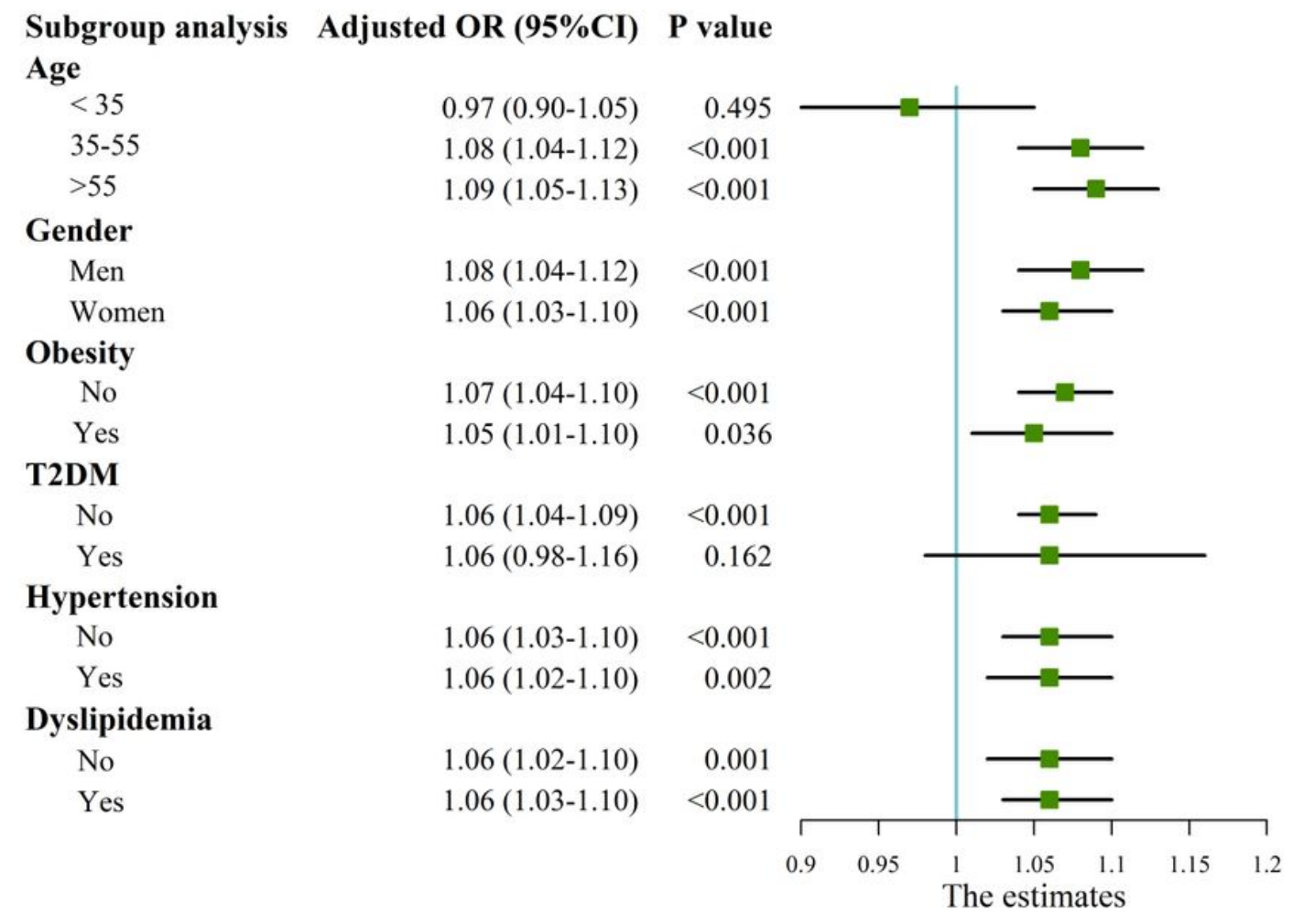

Napping (each $30 \mathrm{~min}$ increment)

Figure 2

Napping (each $30 \mathrm{~min}$ increment) and prevalence of hyperuricemia, stratified by age, gender, and chronic disease conditions. Adjusted for age, gender, education level, marital status, average monthly income, smoking status, drinking status, physical activity, dietary pattern, snoring, night sleep duration, family history of gout, obesity, T2DM, hypertension and dyslipidemia conditions. Each group adjusted for the other covariates except itself. OR= Odds Ratio, $\mathrm{Cl}=$ confidence interval, T2DM = type 2 diabetes mellitus. 
A

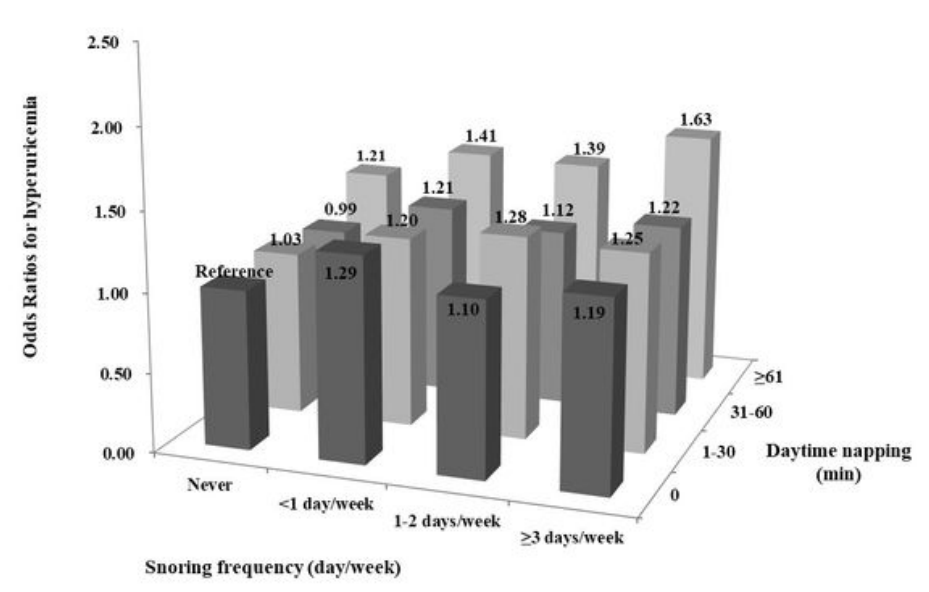

B

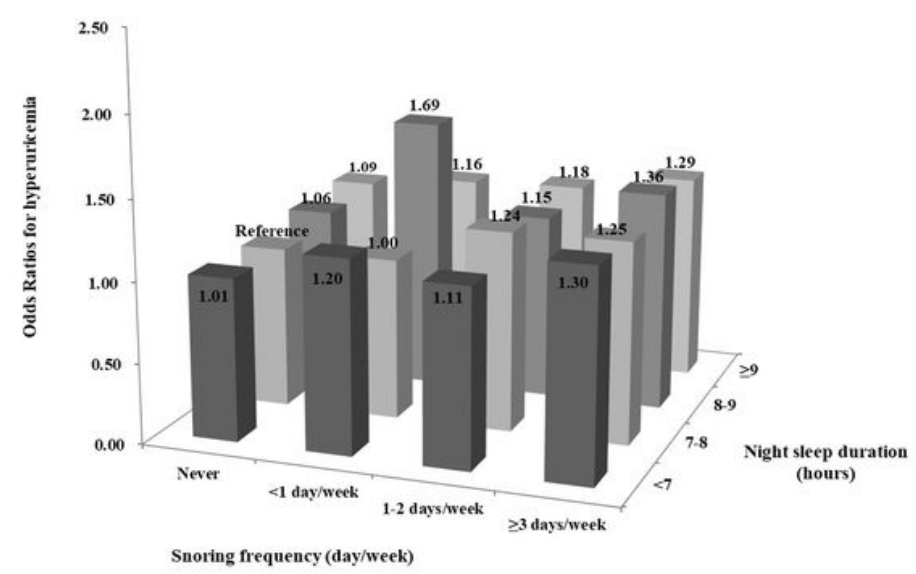

$\mathrm{C}$

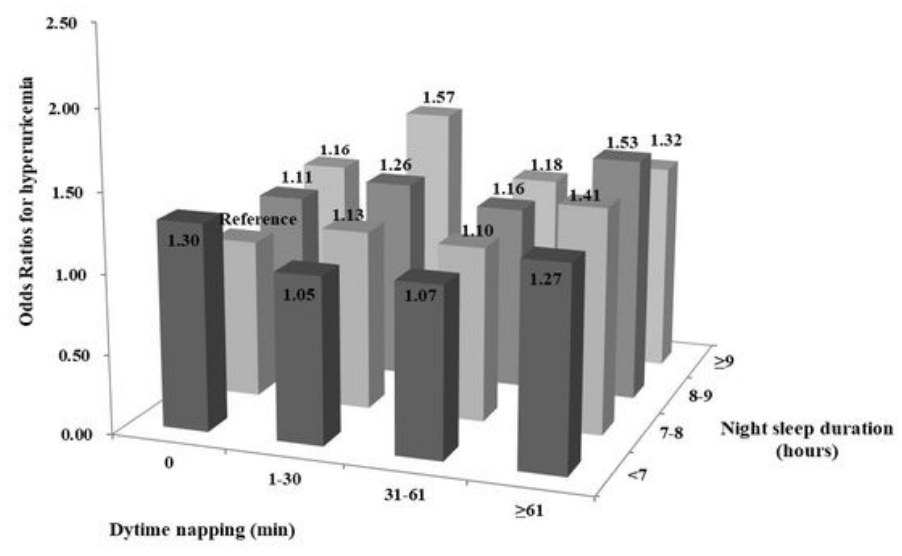

\section{Figure 3}

Joint effects of sleep variables on hyperuricemia. All odds ratios were calculated with joint analyses of snoring frequency and daytime napping $(A)$, snoring frequency and night sleep duration (B), and daytime napping and night sleep duration (C), respectively. Adjusted for age, gender, education level, marital status, average monthly income, smoking status, drinking status, physical activity, dietary pattern, night sleep duration, family history of gout, obesity, T2DM, hypertension and dyslipidemia conditions.

\section{Supplementary Files}

This is a list of supplementary files associated with this preprint. Click to download.

- Additionalfile1.docx 